\title{
Uretroplastia bulbar con injerto: Una actualización sobre las diferentes técnicas quirúrgicas
}

\section{Graft Urethroplasty for Bulbar Strictures: An Update on the Different Surgical Techniques}

\author{
Eduar S. Rodríguez ${ }^{1}$ (1) Laura A. Serna ${ }^{2}$ Wilmer A. Agressot ${ }^{3}$ \\ ${ }^{1}$ Residente de Urología, Universidad El Bosque, Bogotá, Colombia \\ 2 Médica General, Universidad El Bosque, Bogotá, Colombia \\ Address for correspondence Eduar S. Rodríguez, MD, Universidad EI \\ Bosque, Carrera 15 n 135-81, Bogotá, Colombia \\ 3 Urólogo, Universidad Militar Nueva Granada, Bogotá, Colombia \\ (e-mail: shellender@hotmail.com).
}

Urol Colomb 2021;30(3):e217-e222.

\section{Resumen \\ Palabras claves \\ - uretroplastia \\ - injerto de mucosa oral \\ - estrechez uretral \\ - colgajo}

\section{Abstract}

En pacientes con estrechez uretral bulbar de una longitud mayor a $2 \mathrm{~cm}$, que no sean candidatos a otras técnicas, se realiza uretroplastia con injerto. Actualmente se emplean diversas técnicas, cada una con ventajas y desventajas propias.

Describir las ventajas y desventajas de las técnicas quirúrgicas empleadas actualmente en la uretroplastia con injerto, así como sus tasas de éxito.

Se hizo una búsqueda en PubMed, ClinicalKey y en ScienceDirect, utilizando las palabras claves: "urethral stricture," "urethroplasty," "oral graft" y "flap." Se utilizaron los estudios más relevantes, tanto originales como revisiones sistemáticas y metaanálisis, en inglés y en español.

Las diferentes técnicas quirúrgicas ofrecen ventajas y desventajas teóricas frente a las otras, aunque las tasas de éxito en todas es cercana al 90\%, sin ser una francamente superior frente a las demás.

La elección de la técnica quirúrgica a realizar depende de las preferencias y experiencia del cirujano, dado que la tasa de éxito para todas las técnicas es similar.

In patients with urethral stricture longer than $2 \mathrm{~cm}$ who are not candidates for other techniques, graft urethroplasty is the most performed surgery. Currently, several techniques are used, each with their own advantages and disadvantages.

Describe the advantages and disadvantages of the surgical techniques currently used in graft urethroplasty, as well as their success rates.

A search was made in PubMed, ClinicalKey and ScienceDirect, using the keywords: "urethral stenosis," "urethroplasty," "oral graft" and "flap." The most relevant original studies, systematic reviews and meta-analyzes were used, both in English and Spanish. received

December 7, 2018

accepted

July 18, 2019
DOI https://doi.org/

$10.1055 / \mathrm{s}-0039-1696697$

ISSN 0120-789X.

elSSN 2027-0119.
(C) 2021. Sociedad Colombiana de Urología. All rights reserved. This is an open access article published by Thieme under the terms of the Creative Commons Attribution-NonDerivative-NonCommercial-License, permitting copying and reproduction so long as the original work is given appropriate credit. Contents may not be used for commercial purposes, or adapted, remixed, transformed or built upon. (https://creativecommons.org/ licenses/by-nc-nd/4.0/)

Thieme Revinter Publicações Ltda., Rua do Matoso 170, Rio de Janeiro, RJ, CEP 20270-135, Brazil 


\section{Keywords}

- urethral stricture

- urethroplasty

- oral graft

- flap
The different surgical techniques offer theoretical advantages compared with the others, although the success rates in all are close to $90 \%$, without being frankly superior compared with the others.

The choice of the surgical technique to be performed depends on the preferences and experience of the surgeon, given that the success rate for all techniques is similar.

\section{Introducción}

Debido a la mayor longitud de la uretra masculina, la estrechez uretral afecta con mayor frecuencia a los hombres, con una presentación en mujeres menor al 1\% de todos los casos. Se estima que uno de cada 300 hombres padecerá de esa enfermedad durante su vida, ${ }^{1}$ aunque su incidencia real es desconocida. No obstante, existe suficiente evidencia de la relación lineal entre ella y la edad, siendo de $5,8 / 100.000$ en comparación a 9,0/100.000, en hombres menores y mayores de 65 años, respectivamente ${ }^{2}$.

La estrechez uretral implica que haya un proceso de espongiofibrosis. Teniendo eso en cuenta, el término "estrechez uretral" sólo puede emplearse cuando se localiza en la uretra anterior. Dado que la uretra posterior no se encuentra rodeada por cuerpo esponjoso, el término apropiado en esa localización es "estenosis uretral”3.

En pacientes con estrechez de la uretra bulbar con una longitud mayor a $2 \mathrm{~cm}$, que no sean candidatos a otras técnicas, se recomienda la uretroplastia con injerto, ${ }^{4}$ incluso en edad avanzada. ${ }^{5}$ Diversas técnicas se han descrito para su realización, muchas de las cuales se utilizan actualmente en Colombia., ${ }^{6,7}$ En la presente revisión se discutirán los beneficios, desventajas y tasas de éxito de cada una de ellas.

Se hizo una búsqueda en PubMed y en ScienceDirect, utilizando las palabras claves: "urethral stricture," "urethroplasty," "oral graft" y "flap." Se incluyeron los estudios más relevantes, tanto originales como revisiones sistemáticas y meta-análisis, en inglés y en español.

\section{Características del Injerto}

Un injerto corresponde a un segmento de tejido aislado y resecado para su colocación en una ubicación corporal diferente. Durante las primeras 48 horas de haber sido reubicado, problems recibirá los nutrientes desde el lecho receptor por simple difusión, proceso denominado imbibición plasmática, porque carece de irrigación propia. A las 72 horas ocurre la inosculación, en la cual los vasos seccionados del injerto comienzan a formar conexiones con los del lecho receptor ("kissing capillaires"). Hacia el quinto día ya ha ocurrido la angiogénesis, aumentando la vascularización del injerto por la neoformación de capilares. ${ }^{8}$

El injerto que se utilice debe poseer una capa epitelial gruesa, una morbilidad mínima en el sitio donante, carecer de folículos pilosos ${ }^{9}$ y ser además fácil de obtener. ${ }^{10}$ Dentro de los tejidos que históricamente se han utilizado se incluyen: mucosa del colon, ${ }^{11}$ vesical, ${ }^{12}$ lingual y bucal, ${ }^{13}$ así como túnica vaginalis, ${ }^{14}$ piel del pene, ${ }^{15}$ del escroto y del periné $^{16} \mathrm{y}$, recientemente, de tejidos obtenidos por ingeniería tisular. ${ }^{17}$

Actualmente, la mucosa oral es el tejido más utilizado para el injerto, ${ }^{18,19}$ a causa de reunir las características ideales enunciadas previamente, sumado a que su ambiente nativo es similar al de la uretra, su capa epitelial gruesa la hace menos propensa a contraerse y su lámina propia delgada facilita la neovascularización. Durante su obtención debe tenerse la precaución de no lesionar el conducto de Stenon, ubicado a nivel del segundo molar superior. ${ }^{8}$

\section{¿Colgajo o Injerto?}

En un estudio prospectivo, que incluyó a 55 pacientes con una media de edad de 37 años, en los que la estrechez era bulbar en el $85 \%$ de los casos y la longitud media de la estrechez era de 5,6-6 cm, se comparó la tasa de éxito de la uretroplastia de ampliación empleando colgajo de piel de pene posterior versus injerto de mucosa oral posterior con una media de seguimiento de 23 meses. La tasa de éxito fue similar en ambos grupos, con un $85,6 \%$ versus $89,9 \%$, respectivamente $(P, 0,05)$, aunque la satisfacción del paciente fue mayor en el grupo de injerto de mucosa oral. El $89 \%$ de esos últimos, recomendaría su procedimiento a otra persona en comparación a un $65 \%$ en el grupo de colgajo de piel. ${ }^{20}$

Barbagli y col., comparó retrospectivamente el resultado obtenido con el uso de injerto de mucosa oral versus injerto de piel de pene, en 210 pacientes llevados a uretroplastia bulbar anastomótica de aumento o uretroplastia bulbar con injerto onlay, empleando alguno de los dos tejidos. En los casos en los que se empleó el injerto de piel de pene siempre fue dorsal, mientras que en los casos en los que se utilizó mucosa oral la mayoría (el 54\%) fue ventral. La tasa de éxito fue del $82,8 \%$ con el uso de injerto de mucosa oral versus $59,6 \%$ con el uso de injerto de piel de pene. ${ }^{21}$

Aunque el uso de colgajo en comparación al injerto ya fue ontroversial debido a resultados contradictorios, estudios recientes favorecen el segundo por su mayor tasa de éxito, menor dificultad para su realización y menor morbilidad. Debe aclararse que el uso de piel de pene se encuentra contraindicado en pacientes con liquen escleroso, debido a que esa puede estar también comprometida por la enfermedad. $^{10}$

Existen diversas técnicas para la uretroplastia de aumento con injerto, en las que la localización de este puede ser 
anterior, lateral o posterior, con un abordaje onlay o inlay. La experiencia del cirujano es determinante en la tasa de éxito del procedimiento, con mejores resultados tras una larga curva de aprendizaje. ${ }^{22}$ A continuación, se revisarán las principales técnicas.

\section{Injerto dorsal onlay de Mucosa Bucal}

Fue descrita por primera vez en 1996 por Barbagli ${ }^{23}$ para la estrechez de la uretra bulbar. Su técnica incluye una incisión perineal, apertura del músculo bulboesponjoso y liberación circunferencial de la uretra bulbar. Se realiza la uretrotomía dorsal a nivel de la zona estrecha. Se sutura el injerto en la túnica albugínea de los cuerpos cavernosos subyacentes, tanto en sus bordes como en su superficie; eso impide la separación del injerto de su lecho por hematoma o seroma, favoreciendo así la neovascularización.

El injerto dorsal posee un mayor soporte estructural por su fijación a los cuerpos cavernosos, que reduce la posibilidad de contracción o saculación. Teniendo en cuenta la disposición más dorsal de la uretra a nivel bulbar, durante la uretrotomía dorsal se secciona un segmento más delgado de cuerpo esponjoso en comparación a la uretrotomía ventral, por lo que el sangrado es menor. Adicionalmente, en caso de una extensión de la estrechez mayor a la prevista, puede emplearse en uretra no bulbar. Si la estrechez es completa, puede realizarse una reconstrucción anastomótica de aumento e incluso combinarse con un injerto ventral, por lo que la versatilidad es otro de sus beneficios. ${ }^{8}$

Dentro de sus desventajas se incluye una mayor disrupción de la microvasculatura por la disección circunferencial de la uretra, que teóricamente aumentaría la falla del injerto. Se ha asociado también a una menor visibilidad cuando la uretroplastia se realiza en la porción proximal de la uretra bulbar. Adicionalmente, cuando la estrechez se extiende a la uretra membranosa, el mayor grosor dorsal del esfínter urinario externo lo hace más propenso a lesionarse si no se realiza una disección cercana al cuerpo esponjoso. ${ }^{8}$

En dos meta-análisis, que en conjunto analizaron 98 estudios que incluían pacientes llevados a uretroplastia con injerto dorsal onlay de mucosa bucal, coincidieron en demostrar una tasa de éxito de un 88,3\%. ${ }^{24,25}$ Estudios recientes reportan una tasa de éxito de hasta un $92,5 \%{ }^{26}$

\section{Injerto dorso-lateral onlay de Mucosa Bucal}

En el año 2009, Kulkarni modifica la técnica de Barbagli ${ }^{27}$ describiendo la colocación del injerto dorso-lateral onlay. La técnica consiste en la incisión perineal, con posterior disección unilateral de la uretra bulbar, disecando esta sólo del lado izquierdo, dejando el músculo bulbo-esponjoso y su tendón central intactos. La uretra permanece unida al cuerpo cavernoso en su lado derecho, manteniendo así la irrigación arterial ipsilateral. Posteriormente, en el lado izquierdo se rota parcialmente la uretra, hasta exponer adecuadamente la zona de estrechez. Se realiza una uretrotomía dorsal siguiendo la extensión de toda la estrechez. Se toma la medida de la misma, para guiar el tamaño del injerto. El borde lateral derecho del injerto se sutura con el borde izquierdo de la uretrotomía y su superficie se fija al cuerpo cavernoso subyacente con puntos simples de vicryl 4-0. Se realiza el paso de la sonda Foley siliconada de 16 FR. Se rota la uretra a su posición normal y se completa el cierre de la misma.

Esa técnica reúne las ventajas ya mencionadas del injerto dorsal onlay, reduciendo además sus desventajas, pues la disección unilateral contribuye a una mayor preservación de la microvasculatura. Su tasa de éxito es del $92 \%{ }^{27}$

\section{Injerto Ventral onlay de Mucosa Bucal}

Se realiza una incisión perineal, seguido por una incisión en la línea media del músculo bulbo-esponjoso sin tener que lateralizar ese respecto al cuerpo esponjoso. Una vez localizada la estrechez, se realiza la incisión ventral sobre el cuerpo esponjoso. Se sutura el colgajo por sus bordes con los de la uretrotomía y se cubre el colgajo con la adventicia del cuerpo esponjoso. Esa última maniobra provee al colgajo de soporte y de un lecho vascular. ${ }^{28}$ La menor disección requerida y una exposición proximal fácil son sus ventajas, mientras que sus desventajas incluyen: mayor sangrado, menor soporte estructural del injerto y menor adaptabilidad. $^{8}$ Su tasa de éxito es del $88 \%-90 \%,{ }^{24,26}$ muy similar a la del injerto dorsal.

\section{Injerto Lateral onlay de Mucosa Bucal}

Descrito también por Barbagli, ${ }^{29}$ es una mezcla de la técnica dorsal y ventral. Tras realizar la incisión en la línea media del músculo bulbo-esponjoso, se realiza una disección unilateral limitada, reparando el músculo y liberando el cuerpo esponjoso de los cuerpos cavernosos. Se realiza a continuación una incisión lateral en el segmento estrecho, colocando posteriormente el injerto en una forma similar al abordaje ventral. Esa técnica permite una mayor preservación de la microvasculatura, que no se afecta en el lado contralateral, y un menor sangrado con una mejor visualización secundaria. De forma similar al injerto ventral, al no fijarse a los cuerpos cavernosos el soporte estructural es menor y de esa forma aumenta el riesgo teórico de contracción o saculación. No obstante, la tasa de éxito es similar a la del injerto ventral o dorsal, aunque la literatura al respecto es escasa. ${ }^{29}$

\section{Injerto Dorsal inlay de Mucosa Bucal}

Técnica introducida en el año 2002 por Asopa, ${ }^{30}$ consiste en un abordaje inicial similar al ventral. Tras la uretrotomía ventral, se realiza una uretrotomía dorsal hasta exponer una zona elíptica de la albugínea subyacente, sobre la cual se dispone el injerto fijándolo a los bordes de la uretrotomía dorsal y a los cuerpos cavernosos por su superficie. Posteriormente, se cierra la uretrotomía ventral en uno o dos planos. La ventaja de ese procedimiento respecto al onlay radica en una menor disección de los tejidos, dado que no es necesaria la movilización excesiva o la liberación circunferencial de la uretra, preservando así la microvasculatura bilateral. Por otra parte, esa menor movilización también se traduce en 
una menor ampliación del calibre de la uretra y en una limitación para el tamaño de la zona en la que se fijará el injerto. La realización de dos uretrotomías podría también relacionarse con un mayor riesgo de sangrado, fístulas u otras complicaciones. Sin embargo, esos riesgos son sólo teóricos y la tasa de éxito no difiere de los anteriores, reportada en un $87 \%-92 \%{ }^{30,31}$

\section{Injerto Dorsal inlay Combinado con Ventral Onlay}

Descrita en el año 2007 por Palminteri, incluye la utilización de un injerto dorsal inlay combinado con uno ventral onlay. Eso aumenta de forma potencial el calibre de la uretra, preservando la microvasculatura. Sus desventajas incluyen: el requerimiento de un mayor tejido de injerto, un mayor riesgo de saculación en el injerto ventral y de sangrado por la realización de dos uretrotomías. La tasa de éxito reportada es del $85 \% .^{32}$

\section{Conclusión}

El injerto de mucosa oral en uretroplastia ha mostrado mejores resultados que otros tejidos y que colgajos. Esa actualización revisa la literatura más relevante hasta el momento sobre las técnicas quirúrgicas más empleadas en la uretroplastia bulbar con injerto. Todas ellas ofrecen tasas de éxito cercanas al $90 \%$, por lo que su elección dependerá de la preferencia y experiencia del cirujano.

Conflicto de Intereses

Los autores declaran no tener ningún conflicto de intereses.

\section{Referencias}

1 Watkin N, Patel P. The diagnosis and management of acquired urethral stricture disease. Surg (United Kingdom) 2017;35(06): 313-323. Doi: 10.1016/j.mpsur.2017.03.006

2 Lazzeri M, Sansalone S, Guazzoni G, Barbagli G. Incidence, Causes, and Complications of Urethral Stricture Disease. Eur Urol Suppl 2016;15:2-6. Doi: 10.1016/j.eursup.2015.10.002

3 Gómez RG, Mundy T, Dubey D, et al. SIU/ICUD Consultation on Urethral Strictures: Pelvic fracture urethral injuries. Urology 2014; 83(3, Suppl):S48-S58. Doi: 10.1016/J.UROLOGY.2013.09.023

4 Dugi DD III, Simhan J, Morey AF. Urethroplasty for Stricture Disease: Contemporary Techniques and Outcomes. Urology 2016;89:12-18. Doi: 10.1016/j.urology.2015.12.012

5 Levy M, Gor RA, Vanni AJ, et al; Trauma and Urologic Reconstructive Network of Surgeons (TURNS). The Impact of Age on Urethroplasty Success. Urology 2017;107:232-238. Doi: 10.1016/j.urology.2017.03.066

6 Toro A, Gómez M, Gaviria J. Manejo de la estenosis de uretra anterior con técnica combinada: reporte de cuatro casos. Urol Colomb 2013; XXII(02):43-48http://www.urologiacolombiana.com/userfiles/file/ agosto13/REVISTA UROLOGIA.rar\#page $=43$

7 Contreras-García R, García-Perdomo HA, Robayo-Ramirez J. Experiencia en el manejo de la estrechez uretral en un centro de tercer nivel en Colombia. Urol Colomb 2017;26(02):98-103. Doi: 10.1016/j.uroco.2016.10.006

8 Levy ME, Elliott SP. Graft Use in Bulbar Urethroplasty. Urol Clin North Am 2017;44(01):39-47. Doi: 10.1016/j.ucl.2016.08.009
9 Wessells H, Angermeier KW, Elliott S, et al. Male Urethral Stricture: American Urological Association Guideline. JUrol 2017;197(01):182-190. Doi: 10.1016/j.juro.2016.07.087

10 Cheng L, Li S, Wang Z, Huang B, Lin J. A brief review on anterior urethral strictures. Asian J Urol 2018;5(02):88-93. Doi: 10.1016/j. ajur.2017.12.005

11 Xu Y-M, Qiao Y, Sa Y-L, Zhang J, Fu Q Song L-J. Urethral reconstruction using colonic mucosa graft for complex strictures. JUrol 2009;182(03):1040-1043. Doi: 10.1016/j.juro.2009.05.030

12 Kinkead TM, Borzi PA, Duffy PG, Ransley PG. Long-term followup of bladder mucosa graft for male urethral reconstruction. JUrol 1994;151(04):1056-1058http://www.ncbi.nlm. nih.gov/pubmed/8126791. Accessed October 15, 2018

13 Barbagli G, Balò S, Sansalone S, Lazzeri M. Dorsal onlay graft bulbar urethroplasty using buccal mucosa. Afr J Urol 2016; 22:5-10. Doi: 10.1016/j.afju.2015.09.003

14 Ashmawy H, Magama PT. Tunica vaginalis free graft urethroplasty: 10 years experience. Afr J Urol 2018;24:37-40. Doi: 10.1016/j. afju.2017.09.008

15 Vyas PR, Roth DR, Perlmutter AD. Experience with free grafts in urethral reconstruction. J Urol 1987;137(03):471-474http://www. ncbi.nlm.nih.gov/pubmed/3546734. Accessed October 15, 2018

16 Jordan GH. Scrotal and perineal flaps for anterior urethral reconstruction. Urol Clin North Am 2002;29(02):411-416, viii. http://www.ncbi.nlm.nih.gov/pubmed/12371232. Accessed October 15,2018

17 Browne BM, Vanni AJ. Use of Alternative Techniques and Grafts in Urethroplasty. Urol Clin North Am 2017;44(01):127-140. Doi: 10.1016/j.ucl.2016.08.003

18 Engel O, Soave A, Rink M, Fisch M. Reconstructive Management with Urethroplasty. Eur Urol Suppl 2016;15:13-16. Doi: 10.1016/ j.eursup.2015.10.004

19 Hagedorn JC, Voelzke BB. Patient Selection for Urethroplasty Technique: Excision and Primary Reanastomosis Versus Graft. Urol Clin North Am 2017;44(01):27-37. Doi: 10.1016/j.ucl.2016.08.007

20 Dubey D, Vijjan V, Kapoor R, et al. Dorsal onlay buccal mucosa versus penile skin flap urethroplasty for anterior urethral strictures: results from a randomized prospective trial. JUrol 2007;178(06):2466-2469. Doi: 10.1016/j.juro.2007.08.010

21 Barbagli G, Guazzoni G, Lazzeri M. One-stage bulbar urethroplasty: retrospective analysis of the results in 375 patients. Eur Urol 2008; 53(04):828-833. Doi: 10.1016/j.eururo.2008.01.041

22 Fossati N, Barbagli G, Larcher A, et al. The Surgical Learning Curve for One-stage Anterior Urethroplasty: A Prospective Singlesurgeon Study. Eur Urol 2016;69(04):686-690. Doi: 10.1016/j. eururo.2015.09.023

23 Barbagli G, Selli C, Tosto A, Palminteri E. Dorsal free graft urethroplasty. JUrol 1996;155(01):123-126. Doi: 10.1016/ S0022-5347(01)66566-2

24 Mangera A, Patterson JMCC, Chapple CR. A systematic review of graft augmentation urethroplasty techniques for the treatment of anterior urethral strictures. Eur Urol 2011;59(05):797-814

25 Morey AF, Watkin N, Shenfeld O, Eltahawy E, Giudice C. SIU/ICUD Consultation on Urethral Strictures: Anterior urethra-primary anastomosis. Urology 2014;83(3, Suppl)S23-S26. Doi: 10.1016/J. UROLOGY.2013.11.007

26 Vasudeva P, Nanda B, Kumar A, Kumar N, Singh H, Kumar R. Dorsal versus ventral onlay buccal mucosal graft urethroplasty for longsegment bulbar urethral stricture: A prospective randomized study. Int J Urol 2015;22(10):967-971

27 Kulkarni S, Barbagli G, Sansalone S, Lazzeri M. One-sided anterior urethroplasty: a new dorsal onlay graft technique. BJU Int 2009; 104(08):1150-1155. Doi: 10.1111/j.1464-410X.2009.08590.x

28 Morey AFMJ, McAninch JW. Technique of harvesting buccal mucosa for urethral reconstruction. JUrol 1996;155(05):1696-1697

29 Barbagli G, Palminteri E, Guazzoni G, Montorsi F, Turini D, Lazzeri M. Bulbar urethroplasty using buccal mucosa grafts placed on the ventral, dorsal or lateral surface of the urethra: are results 
affected by the surgical technique? JUrol 2005;174(03):955-957, discussion 957-958

30 Asopa HS, Garg M, Singhal GG, Singh L, Asopa J, Nischal A. Dorsal free graft urethroplasty for urethral stricture by ventral sagittal urethrotomy approach. Urology 2001;58(05):657-659

31 Aldaqadossi H, El Gamal S, El-Nadey M, El Gamal O, Radwan M, Gaber M. Dorsal onlay (Barbagli technique) versus dorsal inlay
(Asopa technique) buccal mucosal graft urethroplasty for anterior urethral stricture: a prospective randomized study. Int J Urol 2014;21(02):185-188. Doi: 10.1111/iju.12235

32 Palminteri E, Berdondini E, Colombo F, Austoni E. Small intestinal submucosa (SIS) graft urethroplasty: short-term results. Eur Urol 2007;51(06):1695-1701, discussion 1701. Doi: 10.1016/j. eururo.2006.12.016 